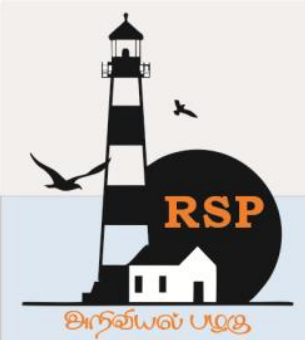

INTERNATIONAL RESEARCH JOURNAL ON ADVANCED SCIENCE HUB

\title{
Basic Design for the development of Autonomous Underwater Vehicle
}

Muniyandy Elangovan ${ }^{1}, T$ Balaji ${ }^{2}$

${ }^{1}$ Professor, Mechanical Department, Vel Tech Rangarajan Dr. Sagunthala R\&D Institute of Science and Technology, Chennai, Tamilnadu, India

${ }^{2}$ Assitant Professor, Mechanical Department, Vel Tech Rangarajan Dr. Sagunthala R\&D Institute of Science and Technology, Chennai, Tamilnadu, India muniyandy.e@gmail.com ${ }^{1}$

\begin{abstract}
The artificial intelligence (AI) is used to automate the operation by taking a decision based on the situation or conditions. It is expected that around 2025, Robotics can move on earth similar to a human and support human society. Artificial Intelligence (AI) is in every field to automate the decision making intelligence to execute the desired operation. Understanding the need and importance of AUV for underwater operation, the authors have identified the need an AUV for the monitor, search and rescue operation. During the development unmanned vehicle, it was identified that the major task and key functionalities to be known to the researchers who want to contribute in this AI domain. The functionalities of AUV is decided by features which can be grouped into six categories as (i) Design of AUV shape (ii) Functionalities (iii) Design of Mechanical and Electrical system (iv) Control system (v) Navigation system (vi) Embedded system/software programming and (vii) Propulsion system. As a researcher, understanding each task or goal is very much needed and useful. Keeping the new researchers need, each task has been studied and highlighted the critical issue in each task which was faced in our development process. This paper can encourage many new researchers to do research in the upcoming domain of AUV.
\end{abstract}

Keywords: $A U V$, artificial intelligence, underwater operation, search, security, robotics

\section{Introduction}

Earth is covered by water which is more than twothird total surface and humans are able to reach or access the underwater less than five-percent [1]. The technology is yet to reach the deep sea even for unmanned underwater vehicles (UUVs). Unmanned Aerial Vehicle (UAV) also equally more needs in commercial as well as defence applications. The UAV was designed and tested for landing and patrolling in the defined area[26] and to travel across one point to another point using GPS and GSM technologies [27] are verified. The robot has been improved to do Butler's service by travelling in the defined path and serve to the defined location. Same time, identify the obstacles and redefine the path for travelling to continue the service [28]. Underwater Unmanned Vehicle is the underwater vehicle that is operated without the need of a human to be present physically in the vehicle. This type can be grouped under two categorized as Remotely Operated Vehicle (ROVs) which is tethered and Autonomous Underwater Vehicles (AUVs).

AUVs are used for mainly military purposes and extended to other field's applications as inspection of underwater structure in Oil and Gas industries/Offshore structures. Also used in an underwater mining operation, mapping of seabed, Intelligence Surveillance and Reconnaissance (ISR), Mine Countermeasures (MCM), AntiSubmarine Warfare (ASW), 


\section{www.rspsciencehub.com}

Inspection/Identification (ID), Oceanography / Hydrography, Communication / Navigation Network Nodes (CN3), Payload Delivery, Influence Activities (IA), Time Critical Strike (TCS), Environmental effects monitoring [10], Seafloor mapping, Geological sampling, Longterm monitoring (e.g. pollution, radiation leakage), Submarine off-board sensing, Water mine search and disposal, Ship hull inspections, Nuclear power plant inspections, Underwater cable inspection. survey of the underwater area for the marine operations $[3,5,7]$ :

AUVs overcome the ROV constraints and are fully automated, capable of performing underwater tasks and missions with the help of an on-board sensor, a navigation system, and payload equipment. AUV can fall in between Mechatronics \& Robotics, and Robot is a subset of Mechatronics [4]. This has been introduced to a student as a freshman engineering which prefers to do a challenging and achievable task in AUV [14]. Tracing systems based on centroid and boundary tracking algorithm was studied 15]. One of the major constraints on the design and development of AUV must be lighter, smaller and cheaper than the currently available AUVs.

\section{Methodology}

AUV must be self-contained (autonomy) for search operation, decision making based on the real-time data or current condition (object detection), intelligent decision (software program) for the immediate surrounding environment or condition is to perform the task or mission (Navigation) and any abnormal condition; vehicles have to come to water surface. This AUV requires an inbuilt autonomous guidance system and control system to carry out the complete operation from the starting point to return to the same place [6], and it can be operated in the range of $50 \mathrm{~km} \mathrm{[10].} \mathrm{Figure} 1$ shows the flow chart to set the order and sequence of solution and combination of solution must be well defined in the program to execute the task by vehicle. This project can be divided into two major parts as one is design and development other systems of AUV, which includes mechanical and electrical system, as well as integration of all subsystems and the second part is modeling and system identification, which includes the required
Volume 02 Issue 11 November 2020

sensor for static and dynamic operation with the simulation of a control system.

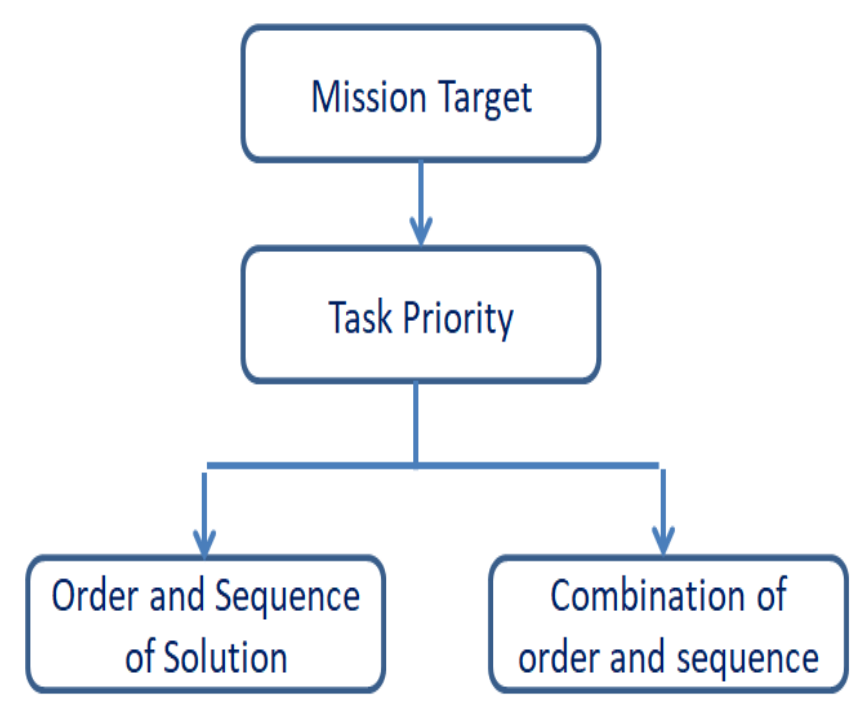

Fig.1. Schematic structure of mission planning

As a first step, design of AUV shape and using potential solver to estimate the forces and motions of the ship under a numerical/computational method. Strip theory [1] and Green function is the potential solver is the reliable solver to estimate the forces and motions of the ship. Hydrodynamics model of an underwater vehicle was studied for the six degrees of freedom and forces, and moments were estimated $[11,12]$.

\section{Design of AUV}

Applications are deciding the shape of the AUV and propulsion system. Based on the targeted goal, the shape of the AUV needs to be finalized, and CFD analysis needs to be carried out to reduce drag, which is proportional to the power requirements for the vehicle [13]. Using CFD, a different marine application was computed to reduce the experimental setup and validated with published results $[17,18,19]$. This study can be extended for the AUV hull to evaluate the hydrodynamic performance, and this study avoids the need for an experimental setup. For any moving vehicle, two coordinate systems are required to track and identify the location easily. One is body fixed and other one is space fixed as shown in Fig2. Lesser the resistance, power consumption is less in underwater operation; power is one of the major constraints. 


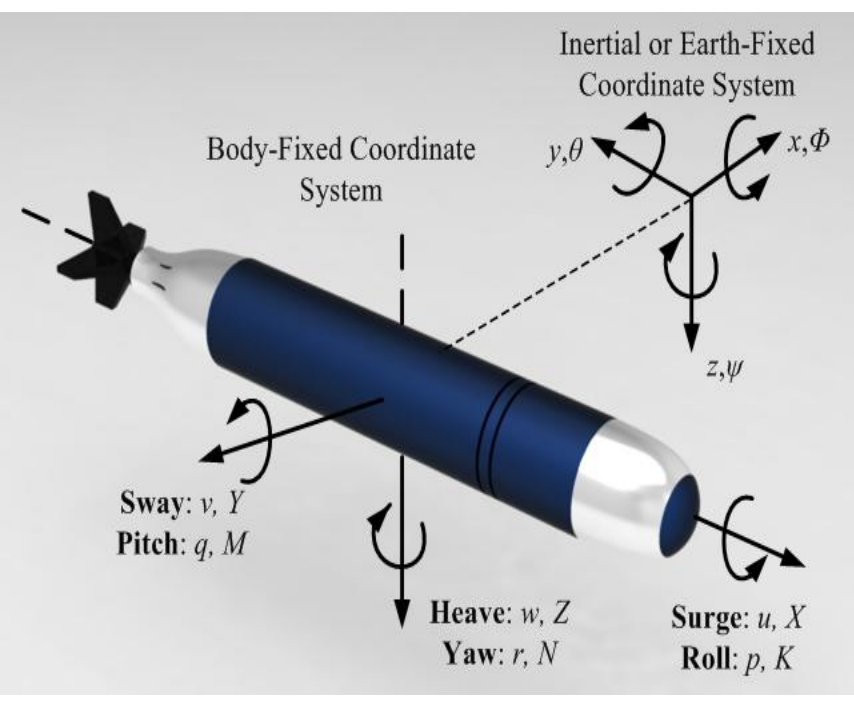

Fig.2. Coordinate system to study UAV motion

The stability of the vehicle is depended on the Buoyancy force and Gravitational force. Ideally, both force needs to have minimum distance, and it must be in a vertically aligned without eccentric, which can cause the moment to the vehicle. Increasing the gravitational force can lead to decrease the roll and pitch motion of the vehicle. Similar to AUV, simplified geometry as the spherical body was studies based on potential theory [22]. The pressure on the vehicle is an addition of atmospheric pressure and static pressure, which is increasing linearly with the depth of water. Initially, watertight hull will have the pressure inside is $1 \mathrm{~atm}$ and that hull surface needs to withstand the static pressure which needs to be considered while designing the wall thickness of the hull.

\section{Basic System and Components}

AUVs system can be grouped as (i) design and development of mechanical and electrical system, (ii) Control system, (iii) Navigation System (iv) Software programming and (v) Propulsion system. In addition to that systems, diving system (ballast tank), Computer microcontroller, sonar system, vision system, depth sensor, watertight hull are required $[2,4]$. Identifying the right instruments/equipment can improve the execution of the task or goal for any projects. From the reader's point of view, Fig 3 shows the major instrument needed in any UAV and additional instruments can be added based on the expected mission.

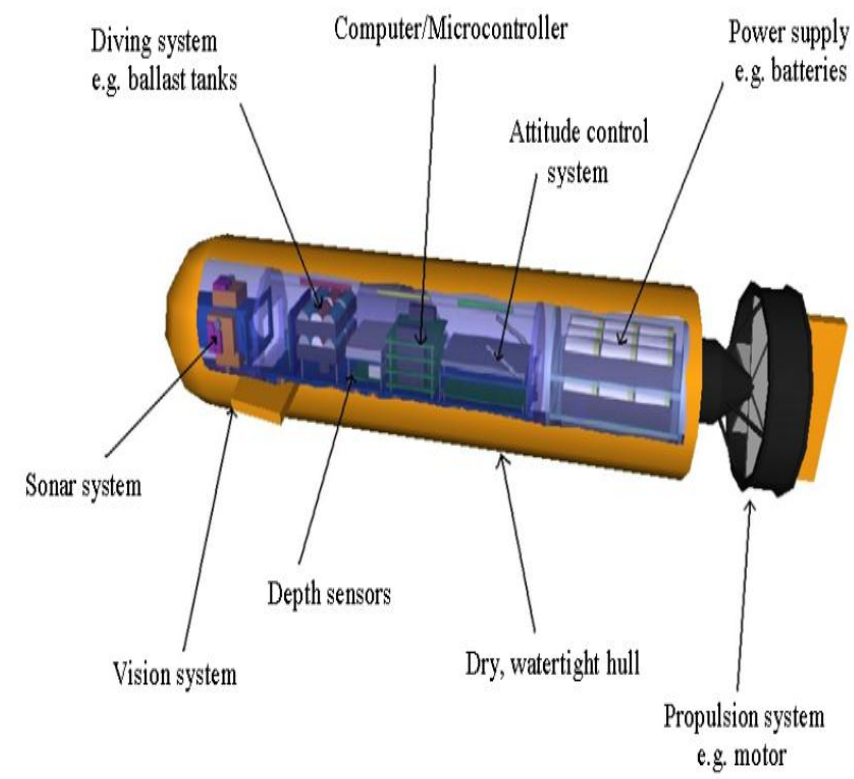

Fig.3. Main instruments needed for AUV (Source: Internet)

\section{Control System}

The control system is one of the key systems in AUV. The control system sent the signal the actuator to achieve the required positions and velocity of the vehicle. The performance of the vehicle depends on the control system architect. While designing the electrical system [2], few needs/data are to be targeted as a primary set for motion control.

- Provide for sonar and vision processing

- Measure the vehicle's depth

- Discern the vehicle's attitude

- Measure the vehicle's surge velocity

- Detect hull breaches and power shortage

- Supply sufficient power for ample testing periods Communication of AUV inside water is another challenge researcher to operate the vehicle, and it has been a highly non-linear behaviour in water, which is more complex to handle the vehicle. Various control systems are used by many researchers, it is proposed to study and adopt the right control system among sliding control, adaptive control, neural networks, and fuzzy logic in different AUVs. To evaluate the performance of the optical detector system by positioning AUV with respect to the light source[16].

\section{Navigation System}

The operation of AUV is depended on knowing the location at all times and greater the locational 
accuracy, reaching the targeted point is easy to complete the assigned task [9]. Doppler velocity $\log$ (DVL) provides the solution to meet the navigation requirements. DVL is small in size and low weight, which is easy to integrate with other navigation systems and the same time range of the seabed to be fixed for the planned operation. The transmit frequency defines the maximum range over the seabed, and the frequency may either be 1000 or $500 \mathrm{kHz}$ [Nortek] respectively $75 \mathrm{~m}$ to 200 $\mathrm{m}$. Pairing the DVL with a high-quality compass can provide the location and speed of the AUV. Navigation system can be grouped into three as (i) Inertial navigation system (INS), acoustic navigation system (ANS) and Geophysical navigation system (GPN) as shown in Fig 4.

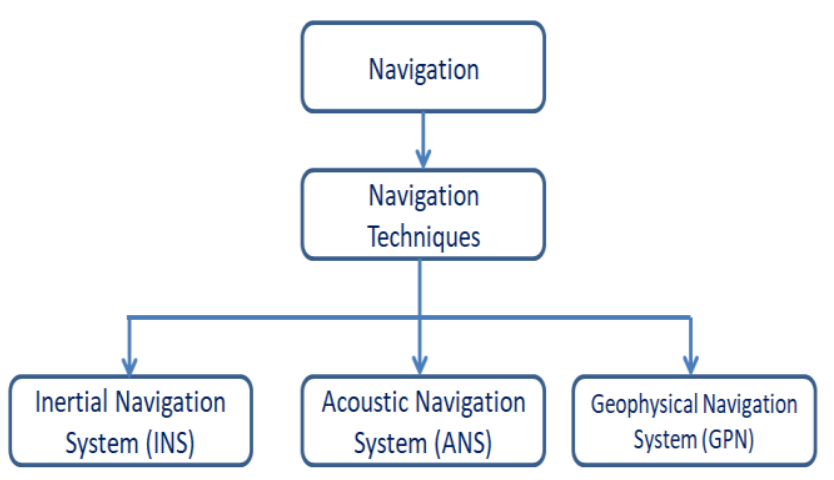

Fig.4. Navigation Techniques

SONAR(Sound Navigation Ranging) is a device that is used to find and identify the object in the water. Sonar has active and passive types where active sonar sends a signal and receive the signal to know the object distance where is passive sonar record or observe the sound. Multibeam sonar is advanced to conventional, which can cover maximum area and side-scan sonar also used in an AUV. The latest development in SONAR is called as Synthetic Aperture Sonar for the mapping and imaging of the seabed with high resolutions [1].

Infrared distance sensors also called as Position Sensing Device, and it is used for mobile detection of wall or obstacles. A digital magnetic compass can deliver the yaw-direction of the AUV with respect to the magnetic field of the earth. The velocity sensor is needed to monitor the vehicle velocity to control the speed within the acceptable limit. An accelerometer is attached to estimate the acceleration of the AUV, and the signal is used to determine the speed and the position of the AUV. Camera can be attached depends on the need to provide clear vision, and it should have the control system to tilt the camera angle to capture very well as upward and downward. The battery is a primary power source, and that is used for the complete operation without any disturbance. To improve the performance of the ship, optimum routing is the one major concept to reduce the voyage time and operational cost of the ship [23]. Optimum route concept can be used for AUV routing because of the consumption of less power achieved by targeting the optimum route to reach the targeted location. For the ship, by changing the trim, fuel consumption can be reduced, and this study was carried for the ship, and it gives around 3 to 4 percent of fuel-saving [25]. Trim curve study can be carried to reduce the resistance which in-turn increases the life of battery power availability.

\section{Propulsion System}

Power is supplied from the Lithium-ion batteries. Supply is connected to the motor which in turn connected with the propeller. All batteries are to be connected in parallel to avoid current flow in between batteries and fuse is recommended to avoid short circuit in the supply line. When selecting the equipment for the AUV, better to have the power consumption low. In the propulsion system, number motor decides the degree of freedom of the vehicle, and it can be reduced of the motor can be controlled to change the direction. B series propeller study was carried out to estimate torque [20] and a similar study can be executed for this vehicle propeller. The position of the motor can cause the vibration in the vehicle, and it is recommended to carry out the interaction between propeller-hull. Thrust is estimated based on the speed of the vehicle as Thrust $=1 / 2 \times$ density $\mathrm{x} \mathrm{v}^{\wedge} 2$ $\mathrm{Cd}$ where $\mathrm{Cd}$ is the drag coefficient [8]. For the thrust power estimation, thrust must be multiplied with velocity. Therefore, maintain the optimum speed play a major role in this otherwise energy consumption will be more for the small task. In underwater operation, keeping energy for longer keeps the vehicle better for better operations. To move the vehicle download against the buoyant force, weight can be increased using ballast or thrusters can be used to move downwards. The weight of the vehicle is played equal to the buoyant force. 


\section{www.rspsciencehub.com}

\section{Conclusions}

As a first step, the AUV hull needs to be designed and tested to keep less resistance using CFD software. To develop an AUV, major tasks and goals are well explained. The complete control system, navigation system, and propulsion systems are to be tested with the specified operation and any additional system can be added as per the targetted functionalities.. An emergency operation must be added to bring back to the original position when there is any abnormal situation arise. Overall, this paper provide a complete idea for the development of AUV and key issues that were discussed. As AUV must be is targeted to achieve operations like search, rescue, monitor and lifting, embedded programming system will be developed to execute all the operation without human interference which brings researcher to compete in this domain for the future generation.

\section{References}

[1] Smith, T.F., Waterman, M.S.: Identification of Common Molecular Subsequences. J. Mol. Biol. 147, 195-197 (1981)

[2] Marcel Valentin, "From Air to Water", The Next Investment Frontier for Drones, Sean Peasgood, 14 September 2015.

[3] Louis Andrew Gonzalez, Thesis "Design, modeling and control of an Autonomous Underwater Vehicle", The University of Western Australia,2004.

[4] Yannick Allard and Elisa Shahbazian, "Unmanned Underwater Vehicle(UUV) information Study", Defense Research and Development Canada, Atlantic Research Centre, 28 November, 2914.

[5] VSB Haranadha Rao, Thesis "Design and Fabrication of underwater hull inspection Vehicle", Vel Tech Rangarajan Dr Sagunthala R\&D Institute of Science and Technology, May 2019.

[6] Bernhard Gerl, Thesis "Development of an Autonomous underwater Vehicle in an Interdisciplinary Context", School of Electrical, Electronic and Computer Engineeringkeeping, The University of Western Australia, 2006.

[7] Somaiyeh Mahmoud Zadeh, Thesis "Autonomous Reactive Mission
Scheduling and Task-Path Planning Architecture for Autonomous Underwater Vehicle", School of Computer Science, Engineering and Mathematics, Faculty of Science and Engineering, Flinders University, Australia, Dec 2016.

[8] Nicolas Marandini, Thesis "Towards sparse seismic acquisition with AUVs: improving underwater navigation and full wavefield migration of seismic and AUV data", Delft University of Technology ETH Zurich RWTH Aachen University, 9 August 2018.

[9] Regardt Busch, Thesis "Modelling and Simulation of an Autonomous Underwater Vehicle", The University of Western Australia School of Electrical, Electronic and Computer Engineering, April 2009.

[10]Khalid M Alzahrani, Thesis "An Underwater Vehicle Navigation System Using Acoustic and Inertial Sensors", Embry-Riddle Aeronautical University, Florida, May 2018.

[11]H. Niu, S. Adams, T. Husain, N. Bose, "Applications of Autonomous Underwater Vehicles in Offshore Petroleum Industry Environmental Effects Monitoring" ,Canadian Institute Of Mining, Metallurgy \& Petroleum Industry Environment Effects Monitoring, 2007-116.

[12]Agus Budiyono, "Advances in unmanned underwater vehicles technologies: Modeling, control and guidance perspectives", Konkuk University, 1 Hwayang-Dong, Seoul 143-701, Korea, 11 September 2009.

[13]Tadahiro Hyakudome, "Design of Autonomous Underwater Vehicle", International Journal of Advanced Robotic Systems, Vol. 8, No. 1 (2011) ISSN 17298806, pp 131-139 Japan.

[14]M Shahrieel et. all, "Design and Development of an Autonomous Underwater Vehicle (AUV-FKEUTeM)", Proceedings of MUCEET2009, Malaysian Technical Universities Conference on Engineering and Technology, Malaysia, June 20-22, 2009.

[15]David Ye et all, "Freshman Project: Autonomous Underwater Vehicle (AUV)", American Society for Engineering 
Education, Polytechnic University, AC 2007-658.

[16]Ryan N et all, "Planning and Implementing Trajectories for Autonomous Underwater Vehicles to Track Evolving Ocean Processes based on Predictions from a Regional Ocean Model", Robotic Embedded Systems Laboratory, University of Southern California, Los Angeles, CA 90089 USA.

[17]Firat Eren et all, "Position, Orientation and Velocity Detection of Unmanned Underwater Vehicles (UUVs) Using an Optical Detector Array", Sensors 2017, 17, 1741; doi:10.3390/s17081741.

[18]Muniyandy ELANGOVAN, "Practical Application of CFD in Marine Industry", International in Shipbuilding and Offshore Engineering (ICSOE 2K18), AMET, Chennai, 23 March 2018.

[19]Muniyandy ELANGOVAN and A.R. Kar, "CFD as an Alternative tool for Marine Experiment", INMARCO-2010, December 9-11, Mumbai, India, 2010.

[20]Muniyandy ELANGOVAN and G. Sahoo, "Generation of Irregular wave fort marine Application by Plunger Type wave Maker", International Symposium on Practical Design of Ships and Other Floating Structures (PRADS-2010), September, 19-24, Rio de Janeiro, Brazil, 2010

[21]G. Sahoo, Anant LAL and Muniyandy ELANGOVAN, "Study and Analysis of B Series Propeller by CFD", 2009 ANSYS India Conference (ANSYS 2009), Pune, India, 16 November, 2009

[22]Muniyandy ELANGOVAN, "Analysis of Hydrodynamic Forces and Motions with high forward speed", Inter. Marine Design Conference (IMDC 2009), Trondheim, Norway, 26-29 May, 2009

[23]Muniyandy ELANGOVAN, "Numerical Analysis of a spherical body in an infinite fluid domain with oscillations by Green Function Method" International conference on Marine Hydrodynamics (MAHY 2006), Naval Science and Technological Lab, Vishakhapatnam, INDIA, January 7-9, 2006
[24]Muniyandy ELANGOVAN and A.R. Indumathi, "Improving the Performance of Existing Ships", International in Shipbuilding and Offshore Engineering (ICSOE 2018), AMET, Chennai, 25-26 Oct 2018 . ISBN: 978-93-85434-72-3.

[25]Muniyandy ELANGOVAN, "Need of cloud Technology and importance of cloud security for maritime industry", National conference on Smart Innovations in Robotics and Cloud Security (NCSIRCS 2017), VIT Chennai, 22 April 2017.

[26]Muniyandy ELANGOVAN, Jagadeesh S, and Jukka, M, "Trim curve generation using CFD for fuel saving", Indian national conference on Applied Mechanics (INCAM), IIT Delhi, 13-15 July 2015.

[27]T. Balaji, D R Gowthaman and R Stalin John "Simple Unmanned Aerial Vehicle Design", International journal of Technology and Engineering System, Volume 5 Issue 2, 2013, PP 18-19.

[28]T. Balaji, R Balamurugan, MR Stalin John and Dr. K. Velmurugan, "Versatile Applied for Agricultural Robot Vehicles", International journal of Automation \& Robotics - IJAMR, Volume 1m Issue 2, 25 June 2014.

[29]T. Balaji, and A T Ravichandran, "Design and Development of SMART robot for butler services", Proceedings of the 2019 2nd International Conference on Service Robotics Technologies, Pages 12-15, Beijing, China - March 22 - 24, 2019

[30]Czajkowski, K., Fitzgerald, S., Foster, I., Kesselman, C.: Grid Information Services for Distributed Resource Sharing. In: 10th IEEE International Symposium on High Performance Distributed Computing, pp. 181-184. IEEE Press, New York (2001)

[31]Foster, I., Kesselman, C., Nick, J., Tuecke, S.: The Physiology of the Grid: an Open Grid Services Architecture for Distributed Systems Integration. Technical report, Global Grid Forum (2002)

[32]National Center for Biotechnology Information, http://www.ncbi.nlm.nih.gov 